\title{
ON A PROBLEM OF E. ČECH ${ }^{1}$
}

\section{J. NOVÅK}

Let $P$ be an abstract set and let $\mathfrak{B}$ be the system of subsets of $P$. An additive, single-valued set-function $u$ taking $\mathfrak{B}$ into $\mathfrak{B}$ and satisfying the conditions $u(0)=0$ and $u(M) \supset M$ for each subset $M$ of $P$ is called a topology in $P$, more precisely, an additive topology ${ }^{2}$ in $P$. The space $P$ with the topology $u$ is denoted by $(P, u)$ or, briefly, $P$. Instead of $u(M)$ we shall write simply $u M$. We define:

$$
u^{0} M=M, u^{1} M=u M, u^{\xi} M=u\left(u^{\xi-1} M\right) \text { for isolated ordinals } \xi,
$$

and

$$
u^{\xi} M=\underset{0 \leq_{\eta}<\xi}{\bigcup} u^{\eta} M \text { for non-isolated } \xi .
$$

The set $M$ is closed if $M=u M . \phi(M)$ is the least ordinal number $\xi$ for which the set $u^{\xi} M$ is closed, that is, $u^{\xi} M=u^{\xi+1} M . G(P, u)$ is the set of all ordinal numbers $\phi(M)$ where $M \subset P$.

E. Cech ${ }^{3}$ has posed the following problem: What are necessary and sufficient conditions on a set $H$ of ordinals in order that there exist a topology $u$ in a countable set $P$ for which $H=G(P, u)$ ? V. Jarník ${ }^{4}$ solved the generalization of the problem for a set of arbitrary cardinal number $\boldsymbol{\aleph}_{\rho}$; his topology, however, is not an additive one, as it satisfies only the axiom of monotony. The present paper contains a solution to this problem when $u$ is to be additive; this solution is stated in Theorem 1. The proof is carried through for a class of cardinals including $\boldsymbol{\aleph}_{0}$.

First we have to prove the following lemma.

LEMma. If the space $(P, u)$ contains a non-closed subset, then there exists a non-closed subset $A \subset P$ such that $u A=u^{2} A$.

Let $M$ be a non-closed subset. The set $u M-M$ is nonvoid: choose $x$ in $u M-M$ and let $A=P-(x)$. By monotony of $u, A \neq u A=P$, so $A$

Received by the editors November $12,1948$.

1 This paper was rewritten for the Bulletin; the transcriber takes full responsibility for any errors that may have been introduced by this process.

${ }^{2}$ Every set function $f$ satisfies the axiom of monotony: If $M \subset N$, then $f(M) \subset f(N)$.

${ }^{3}$ E. Cech, Topologické prostory, Casopis pro Pestován Matematiky a Fysiki vol. 66 (1937) pp. D225-D264, p. D264.

4 V. Jarnik, Sur un probleme de M. Cech, Vexstnik královské đeské společnosti nauk (1938) pp. 1-7. 
is nonclosed but $u A$ is closed. ${ }^{5}$

TheOREM 1. Let $H$ be a set of ordinal numbers. There exists an additive topology $u$ in a countable set $P$ (different from $u M=M$ for all $M \subset P)$ for which $H=G(P, u)$ if and only if: ${ }^{6}$

$1^{\circ}$. $H$ contains only countable ordinals (that is, ordinals of the first and second number classes) including 0 and 1 , and

$2^{\circ}$. If $\alpha$ and $\beta$ are ordinal numbers such that $\alpha+\beta \in H$, then $\beta \in H$.

Necessity. Obviously $0 \in H$; by the lemma, 1 also is in $H$. Every $\phi(M)$ is countable since $P$ can contain no uncountable ascending family of distinct sets. ${ }^{7} 2^{\circ}$ follows from the equality $u^{\xi+\beta} M=u^{\xi+\beta+1} M$, which can be rewritten as $u^{\beta}\left(u^{\xi} M\right)=u^{\beta+1}\left(u^{\xi} M\right)$. (If $P$ is an arbitrary set of cardinal $\boldsymbol{\aleph}_{\rho}$, the same necessity proof works with $1^{\circ}$ changed to allow only ordinals corresponding to cardinals not greater than $\boldsymbol{N}_{\rho_{0}}$ )

Sufficiency. This proof will be carried through for cardinals $\boldsymbol{\aleph}_{p}$ satisfying the following condition: If $R$ is a set of cardinal number $\boldsymbol{\aleph}_{\rho}$, there exists a family $\mathfrak{S}$ of cardinal number $\boldsymbol{\aleph}_{\rho+1}$ whose elements are subsets $P_{\lambda}$ of $R, \lambda<\omega_{\rho+1}$, such that each $P_{\lambda}$ is of cardinal $\aleph_{p}$ while

$$
P_{\lambda} \cap P_{\mu}, \quad \lambda \neq \mu \text {, has cardinal less than } \aleph_{\rho} .
$$

Such cardinals do exist; in particular, taking $R$ to be the set of rational numbers, well-ordering the irrationals, and defining $P_{\lambda}$ to be a sequence of rationals converging to the $\lambda$ th irrational, shows that $\boldsymbol{N}_{0}$ is such a cardinal. Define $M \sim 0$ to mean that the cardinal of $M<\boldsymbol{\aleph}_{\text {p. }}$.

We assume that $H$ is a set of ordinals satisfying $1^{\circ}$, generalized to allow ordinals less than $\omega_{p+1}$, and $2^{\circ}$. To topologize a set $R$ of the given cardinal $\aleph_{\rho}$, assume that $H$ is well-ordered by magnitude, $H=\alpha_{0}$, $\alpha_{1}, \cdots, \alpha_{\lambda}, \cdots$, with $\alpha_{\lambda}<\omega_{\rho+1}$ and $\lambda<\omega_{\sigma}, \sigma \leqq \rho+1$. Then, by $1^{\circ}$, $\alpha_{0}=0$ and $\alpha_{1}=1$. For each $\lambda<\omega_{\sigma}$ and each $\xi \leqq \alpha_{\lambda}$ let $P_{\lambda \xi}$ be subsets of $P_{\lambda}$ such that

$$
\begin{aligned}
& P_{\lambda 0} \subset P_{\lambda 1} \subset \cdots \subset P_{\lambda \xi} \subset \cdots \subset P_{\lambda \alpha_{\lambda}}, \\
& P_{\gamma \xi} \text { and } P_{\lambda \xi+1}-P_{\lambda \xi} \text { are of cardinal number } \aleph_{\rho,}
\end{aligned}
$$

and

$$
P_{\lambda \alpha_{\lambda}}=P_{\lambda} \text { and } P_{\lambda \xi}=\underset{0 \leqq \eta<\xi}{\bigcup} P_{\lambda \eta} \text { for non-isolated } \xi \text {. }
$$

- By footnote $2, u$ is a monotone.

- The condition $2^{\circ}$ is essentially the same as Jarnik's condition 3 in the paper cited under footnote 4. $1 \in H$.

V. Jarnik defines $\phi(0)=\phi(P)=1$; therefore according to Jarnik ex definitione 
Set $P_{\lambda-1}=0$ and assume for convenience that -1 is less than all ordinals.

For each subset $M$ of $R$ and each $\lambda<\omega_{\theta}$ define the symbol $(\lambda M)$ to be the least index $\xi$ such that $M \cap\left(P_{\lambda}-P_{\lambda \xi}\right) \sim 0$. Then $-1 \leqq(\lambda M)$ $\leqq \alpha_{\lambda}$, and $(\lambda M)=-1$ if and only if $M \cap P_{\lambda} \sim 0$. We now prove

$$
(\lambda[M \cup N])=\max [(\lambda M),(\lambda N)] .
$$

Indeed it follows from the definition that $(\lambda A) \leqq(\lambda B)$ if $A \subset B$. Therefore $(\lambda[M \cup N]) \geqq \max [(\lambda M),(\lambda N)]$. Suppose that, on the other hand, $(\lambda M) \leqq(\lambda N)$; then the set $(M \cup N) \cap\left(P_{\lambda}-P_{\lambda(\lambda N)}\right) \sim 0$ so that $(\lambda[M \cup N]) \leqq \max [(\lambda M),(\lambda N)]$.

For each $\lambda<\omega_{\sigma}$ define $f_{\lambda}(\xi)=\xi+1$ for $0 \leqq \xi<\alpha_{\lambda}, f_{\lambda}(-1)=-1$, and $f_{\lambda}\left(\alpha_{\lambda}\right)=\alpha_{\lambda}$. Say that a subset $M$ of $R$ is of kind $\alpha$ if there exist a finite set of indices, $\lambda_{1}, \cdots, \lambda_{n}$, such that $M-U_{i \leqq n} P_{\lambda_{i}} \sim 0$; otherwise say that $M$ is of kind $\beta$. If $F$ is a set of kind $\alpha$, put

$$
F(M)=\bigcup_{i \leqq n} P_{i f_{i}\left(\lambda_{i} M\right)},
$$

where, for brevity, $P_{\lambda_{i}}$ and $f_{\lambda_{i}}$ have been replaced by $P_{i}$ and $f_{i}$. If $M$ is of kind $\alpha$, define $u M=M \cup F(M)$; if $M$ is of kind $\beta$, define $u M=R$.

If $M$ is of kind $\alpha$, the set $F(M)$ is uniquely defined. Indeed, let $\bigcup_{i \leqq n} P_{\lambda_{i}} \supset M \subset \bigcup_{j \leqq m} P_{\mu_{j}}$ and take $i \leqq n$; then $M \cap P_{\lambda_{i}}-U_{j \leqq m} P_{\mu_{j}} \sim 0$. By (1) either $\lambda_{i}$ is a $\mu_{j}$ or $\left(\lambda_{i} M\right)=-1$ and $P_{i f_{i}\left(\lambda_{i} M\right)}=0$. Hence every contribution to $F(M)$ by a $\lambda_{i}$ is supplied by a $\mu_{j}$, and conversely.

$M \cup N$ is of kind $\alpha$ if and only if both $M$ and $N$ are of kind $\alpha$. Also for sets of kind $\alpha$ we have $F(M \cup N)=F(M) \cup F(N)$. Indeed, let $M \cup N-U_{i \leqq n} P_{\lambda_{i}} \sim 0$. By (5) the contribution, $P_{i f_{i}\left(\lambda_{i}[M \cup N]\right) \text {, of the }}$ $i$ th term to $F(M \cup N)$ comes either from $F(M)$ or $F(N)$. From this it follows that $u$ is an additive topology.

First, $u$ is uniquely defined for each subset of $R$ and $M \subset u M$; also $u 0=0$ since the void set is of kind $\alpha . u$ is additive for sets of kind $\alpha$ for $u(M \cup N)=F(M \cup N) \cup M \cup N=F(M) \cup F(N) \cup M \cup N=u M \cup u N$. Every set $M \sim 0$ is of kind $\alpha$ and $F(M)=0$ so $u M=M$ if $M \sim 0$. If either $M$ or $N$ is of kind $\beta, u[M \cup N]=R=u M \cup u N$.

Since $\left(\lambda P_{\lambda \xi}\right)=\xi$, (3) and (4) imply that $u P_{\lambda \xi}=P_{\lambda \xi+1}$ for $0 \leqq \xi<\alpha_{\lambda}$ and $u P_{\lambda \xi}=P_{\lambda}$ for $\xi=\alpha_{\lambda}$. By transfinite induction the reader can easily verify from this and (4) that

(6) $u^{\eta} P_{\lambda \xi}=P_{\lambda \xi+\eta}$ for $\xi+\eta \leqq \alpha_{\lambda} ; \quad u^{\eta} P_{\lambda \xi}=P_{\lambda}$ for $\xi+\eta \geqq \alpha_{\lambda}$.

It remains to show that $G(R, u)=H$. By (6) with $\xi=0, \eta=\alpha_{\lambda}$, it follows that $\phi\left(P_{\lambda_{0}}\right)=\alpha_{\lambda}$. Hence $H \subset G(R, u)$. To establish the opposite 
inequality we must prove $\phi(M) \in H$ if $M \subset R$. If $u M=u^{2} M, \phi(M)=0$ or 1 , so it is in $H$ by $1^{\circ}$. If $u M \neq u^{2} M$, then $M$ is of kind $\alpha$, for $u M=R$ $=u^{2} M$ if $M$ is of kind $\beta$. We note also that if $u A=u B$ and $u A \neq u^{2} A$, from the equalities $u^{\eta} A=u^{\eta} B$, it follows that $\phi(A)=\phi(B)$. Hence for $M-U_{i \geqq n} P_{\lambda_{i}} \sim 0$ we have

$$
u M=\bigcup_{i \leqq n} P_{i f_{i}\left(\lambda_{i} M\right)} \cup K=u\left(\bigcup_{i \leq n} P_{i\left(\lambda_{i} M\right)} \cup K\right),
$$

where $K \sim 0$. By the remarks above, since $u M \not u^{2} M$, we have

$$
\phi M=\phi\left(\bigcup_{i \leqq n} P_{i\left(\lambda_{i} M\right)} \cup K\right) .
$$

By (6) and additivity of $u, \phi(M)=\max _{i \leqq n} \phi\left(P_{i\left(\lambda_{i} M\right)}\right)$ so it suffices to see that every $\phi\left(P_{\lambda_{\xi}}\right)$ is in $H$. However if $\eta=\phi\left(P_{\lambda_{\xi}}\right)$, then by (6) $\xi+\eta=\phi\left(P_{\lambda_{0}}\right)=\alpha_{\lambda} \in H$; by $2^{\circ}, \eta \in H$. This completes the proof.

Brno, Czechoslovakia 